\title{
HEAVY FLAVOR, QUARKONIUM PRODUCTION AND DECAY
}

\author{
XIAOYAN SHEN \\ Institute of High Energy Physics, Chinese Academy of Science \\ Beijing 100049, P. R. China \\ E-mail: shenxy@ihep.ac.cn
}

\begin{abstract}
Recent experimental results on quarkonium physics are reviewed. In particular, the new observed particles since last one or two years, such as $X(1835), X(3872), X(3940), Y(3940)$ and $Y(4260)$ are discussed, the latest data on double charmonium production, heavy hadron spectroscopy and quarkonia decays are presented.
\end{abstract}

\section{Introduction}

The simplest QCD potential, the so called "Cornell" potential, can be written as:

$$
V(r)=-\frac{4}{3} \frac{\dot{\alpha_{s}}}{r}+k \dot{r}
$$

The first term describes the one-gluon exchange, dominating at short distances $(<0.1$ $\mathrm{fm})$ with large momentum transfers. This is the asymptotically "free" regime where the $\alpha_{s}$ is small and the perturbative calculations can be performed. The second term, important at large distances $(>1 \mathrm{fm})$ with low momentum transfers, leads to the "confinement" and is in a regime where the $\alpha_{s}$ is large, making the calculation non-perturbative.

QCD has been tested extensively at the high momentum transfer by lots of high precision experiments. At low energy, it is difficult to be tested due to the non-perturbative nature. Quarkonia, the bound states of quark and its antiquark, are the QCD equivalents of positronium $\left(e^{+} e^{-}\right)$in QED. Quarkonia form the simplest strongly interacting systems with only two constituents (unlike baryons) and identical flavor (unlike mesons with "open" flavor). Light quarkonia are highly relativistic. They also contain mixtures of quarks of different flavors and so can be easily fall apart into other mesons. Charmonium $(c \bar{c})$ is the first heavy quarkonium discovered and is less relativistic. Bottomonium $(b \bar{b})$ is heavier, therefore is less non- relativistic and has a large number of longlived states. The toponium system would have been completely non-relativistic. However, the weak decays will be dominant over the strong decays in such systems. Therefore, charmonium and bottomonium play a special role in probing the strong interactions. The properties of charmonia and bottomonia, and their productions and decays are good labs. for QCD in both perturbative and nonperturbative regimes.

Firstly, the new observations of $X(1835)$, $X(3940), Y(3940)$ and $Y(4260)$ are reported. Then, the latest data on heavy quarkonium production, in particular the big discrepancy on double charmonium production between data and theory are presented. Recent experimental heavy hadron spectroscopy results, including the results from $\eta_{c}^{\prime}, h_{c}$ and $X(3872)$ are reviewed. Also reported are some latest experimental results in quarkonium decays, such as the production of $\sigma$ and $\kappa$, as well as the non- $D \bar{D}$ decays of $\psi(3770)$.

\section{New observations}

There are long-standing predictions of baryonium states ${ }^{1}$, multi-quark states, $q \bar{q}$-gluon hybrids and glueballs. These states have been searched for by many experiments for many years. However, none of them is identified after all the efforts. Recently there has been a revival of interest in the possible existence of the states of non- $q \bar{q}$ or non- $q q q$. Reported 
below are the new observations in searching for such states.

\subsection{The observation of $X(1835)$ in $J / \psi \rightarrow \gamma \eta^{\prime} \pi^{+} \pi^{-}$at BESII}

An anomalous enhancement near the mass threshold in the $p \bar{p}$ invariant mass spectrum from $J / \psi \rightarrow \gamma p \bar{p}$ decays was reported by the BESII experiment ${ }^{2}$. This enhancement was fitted with a sub-threshold $S$-wave Breit-Wigner resonance function with a mass $M=1859_{-10-25}^{+3+5} \mathrm{MeV} / \mathrm{c}^{2}$, a width $\Gamma<$ $30 \mathrm{MeV} / \mathrm{c}^{2}$ (at the $90 \%$ C.L.) and a product branching fraction (BF) $B(J / \psi \rightarrow \gamma X)$. $B(X \rightarrow p \bar{p})=\left(7.0 \pm 0.4(\text { stat })_{-0.8}^{+1.9}(\right.$ syst $\left.)\right) \times$ $10^{-5}$. This surprising experimental observation has stimulated a number of theoretical speculations ${ }^{3,4,5,6,7,8}$ and motivated the subsequent experimental observation of a strong $p \bar{\Lambda}$ mass threshold enhancement in $J / \psi \rightarrow$ $p K^{-} \bar{\Lambda}$ decay $^{9}$. Among various theoretical interpretations of the $p \bar{p}$ mass threshold enhancement, the most intriguing one is that of a $p \bar{p}$ bound state (or barionium) ${ }^{3,1,6}$, which has been the subject of many experimental searches ${ }^{10}$.

If such a structure is interpreted as a $p \bar{p}$ bound states, it is desirable to observe this state in other decay modes. Possible decay modes for a $p \bar{p}$ bound state, suggested in Ref. ${ }^{5,6}$, include $\pi^{+} \pi^{-} \eta^{\prime}$.

The $J / \psi \rightarrow \gamma \pi^{+} \pi^{-} \eta^{\prime}$ decay, with $\eta^{\prime}$ being tagged in its two decay modes, $\eta^{\prime} \rightarrow$ $\pi^{+} \pi^{-} \eta(\eta \rightarrow \gamma \gamma)$ and $\eta^{\prime} \rightarrow \gamma \rho$, is analyzed based on $5.8 \times 10^{7} \mathrm{~J} / \psi$ events collected at BESII.

Figure 1(a) shows the $\pi^{+} \pi^{-} \eta$ invariant mass distribution. The $\eta^{\prime}$ signal is clearly seen. The $\pi^{+} \pi^{-} \eta^{\prime}$ invariant mass spectrum for the selected events is shown in Fig. 1(b), where a peak at a mass around $1835 \mathrm{MeV} / \mathrm{c}^{2}$ is observed (named $X(1835)$ ). The $\gamma \rho$ invariant mass distribution also shows a clear $\eta^{\prime}$ signal (Fig. 1 (c)). A peak near $1835 \mathrm{MeV} / \mathrm{c}^{2}$ is evident in the $\pi^{+} \pi^{-} \eta^{\prime}$ invariant mass spec-
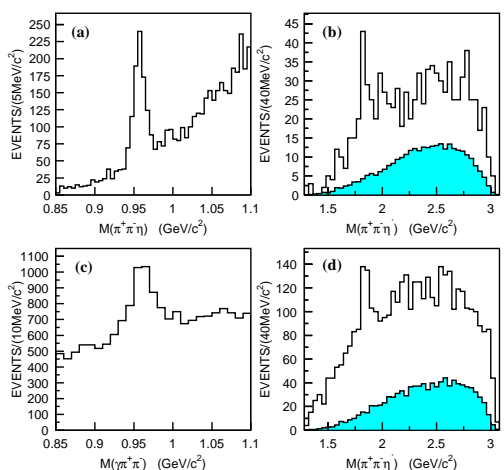

Figure 1. Invariant mass distributions for selected $J / \psi \rightarrow \gamma \pi^{+} \pi^{-} \eta^{\prime}$ candidate events: (a) The $\pi^{+} \pi^{-} \eta$ invariant mass distribution. (b) The $\pi^{+} \pi^{-} \eta^{\prime}$ invariant mass distribution with $\eta^{\prime} \rightarrow \pi^{+} \pi^{-} \eta$. (c) The $\gamma \rho$ invariant mass distribution. (d) The $\pi^{+} \pi^{-} \eta^{\prime}$ invariant mass distribution with $\eta^{\prime} \rightarrow \gamma \rho$. The open histograms are data and the shaded histograms represent $J / \psi \rightarrow \gamma \pi^{+} \pi^{-} \eta^{\prime}$ phase-space MC events (with arbitrary normalization).

trum (Fig. 1 (d)).

The combined $\pi^{+} \pi^{-} \eta^{\prime}$ spectrum with $\left(\eta^{\prime} \rightarrow \pi^{+} \pi^{-} \eta\right)$ and $\left(\eta^{\prime} \rightarrow \gamma \rho\right)$ is fitted with a Breit-Wigner (BW) function convoluted with a Gaussian mass resolution function and a smooth polynomial background function. The BW mass and width of $X(1835)$ obtained from the fit (shown in Fig. 2) are $M=1833.7 \pm 6.1 \mathrm{MeV} / \mathrm{c}^{2}$ and $\Gamma=67.7 \pm 20.3$ $\mathrm{MeV} / \mathrm{c}^{2}$, respectively. The statistical significance for the signal is $7.7 \sigma$. The mass and width of the $X(1835)$ are not consistent with any known particle ${ }^{11}$. The product branching fractions is determined to be

$$
\begin{aligned}
B(J / \psi \rightarrow & \gamma X(1835)) \cdot B\left(X(1835) \rightarrow \pi^{+} \pi^{-} \eta^{\prime}\right) \\
& =(2.2 \pm 0.4 \pm 0.4) \times 10^{-4}
\end{aligned}
$$

The measured $X(1835)$ mass is consistent with the mass obtained from the $J / \psi \rightarrow \gamma p \bar{p}$ channel, while the width is higher by $1.9 \sigma$ than the upper limit on the width reported in Ref. ${ }^{2}$. However, the refitted mass and width of $p \bar{p}$ threshold enhancement, after including the final state interaction effect, are consistent with those of $X(1835)$. 


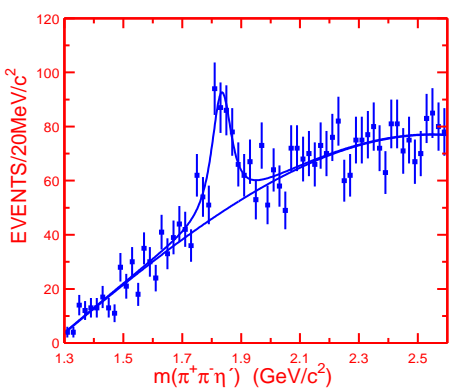

Figure 2. The $\pi^{+} \pi^{-} \eta^{\prime}$ invariant mass distribution for selected events from the $J / \psi \rightarrow \gamma \pi^{+} \pi^{-} \eta^{\prime}\left(\eta^{\prime} \rightarrow\right.$ $\left.\pi^{+} \pi^{-} \eta, \eta \rightarrow \gamma \gamma\right)$ and $J / \psi \rightarrow \pi^{+} \pi^{-} \eta^{\prime}\left(\eta^{\prime} \rightarrow \gamma \rho\right)$ analyses. The solid curve is the fit and the dashed curve indicates the background function.

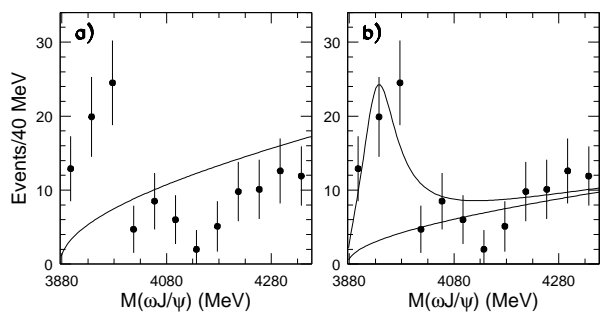

Figure 3. $B \rightarrow K \omega J / \psi$ signal yields vs $M(\omega J / \psi)$. The curve in (a) indicates the result of a fit that includes only a phase-space-like threshold function. The curve in (b) shows the result of a fit that includes an $S$-wave Breit-Wigner resonance term.

\subsection{Near $\omega J / \psi$ threshold enhancement $Y(3940)$ in $B \rightarrow K \omega J / \psi$ at Belle}

$B$ meson decays are a prolific source of $c \bar{c}$ pairs and the large $B$ meson samples produced at $B$-factories are providing opportunities to search for missing $c \bar{c}$ charmonium mesons as well as more complex states.

Based on a $253 \mathrm{fb}^{-1}$ data sample that contains 275 million $B \bar{B}$ pairs collected with the Belle detector, a study of the $\omega J / \psi$ system produced in exclusive $B \rightarrow K \omega J / \psi$ decays is performed.

The $B$-meson signal yields from the binned one-dimensional fits to the $M_{b c}$ and $\Delta E$ distributions for events in different $M(\omega J / \psi)$ intervals are plotted in Figs. 3(a) and (b). Here, $M_{b c}$, the beam constrained mass, is equal to $\sqrt{E_{\text {beam }}^{2}-p_{B}^{2}}$ and $\Delta E$, the energy difference, is $E_{\text {beam }}-E_{B}$, with $E_{\text {beam }}$ representing the cms beam energy, $p_{B}$ the vector sum of the cms momenta of the $B$ meson decay products and $E_{B}$ their cms energy sum.

An enhancement, denoted as $Y(3940)$, is evident around $M(\omega J / \psi)=3940 \mathrm{MeV}$. The curve in Fig. 3(a) is the result of a fit with a threshold function of the form $f(M)=$ $A_{0} q^{*}(M)$, where $q^{*}(M)$ is the momentum of the daughter particles in the $\omega J / \psi$ rest frame. This functional form accurately reproduces the threshold behavior of Monte Carlo simulated $B \rightarrow K \omega J / \psi$ events that are generated uniformly distributed over phasespace. The fit quality to the observed data points is poor $\left(\chi^{2} /\right.$ d.o.f. $\left.=115 / 11\right)$, indicating a significant deviation from phase-space.

Figure. 3(b) shows the results of a fit where an $S$-wave Breit-Wigner (BW) function is included to represent the enhancement. The fit, which has $\chi^{2} /$ d.o.f. $=15.6 / 8$ $(\mathrm{CL}=4.8 \%)$, yields the mass and width of the signal to be $M=3943 \pm 11 \pm 13 \mathrm{MeV}$ and $\Gamma=87 \pm 22 \pm 26 \mathrm{MeV}$. The statistical significance of $Y(3940)$ is $8.1 \sigma$ and the product branching fraction is

$$
\begin{aligned}
\mathcal{B}(B \rightarrow & K Y(3940)) \mathcal{B}(Y(3940) \rightarrow \omega J / \psi) \\
& =(7.1 \pm 1.3 \pm 3.1) \times 10^{-5}
\end{aligned}
$$

This $Y(3940)$ peaks above $D \bar{D}^{*}$. It is expected that a $c \bar{c}$ charmonium meson with this mass would dominantly decay to $D \bar{D}$ and/or $D \bar{D}^{*}$. While for the $c \bar{c}$-gluon hybrid charmonium states, which were first predicted in $1978^{12}$ and are expected to be produced in $B$ meson decays ${ }^{13}$, their decays to $D^{(*)} \bar{D}^{(*)}$ meson pairs are forbidden or suppressed. Therefore, whether $Y(3940)$ can decay to $D \bar{D}$ and/or $D \bar{D}^{*}$ or not is crucial to identify its being a hybrid charmonium or a conventional charmonium state. 


\subsection{Observation of $X(3940)$ in $e^{+} e^{-}$ annihilation at $\sqrt{s} \approx 10.6 \quad \mathrm{GeV}$ at Belle}

The double charmonium production process of $e^{+} e^{-} \rightarrow J / \psi+X$ is investigated using the integrated luminosity of $350 \mathrm{fb}^{-1}$ data sample collected by the Belle detector at the $\Upsilon(4 S)$ resonance and nearby continuum. The $J / \psi$ is reconstructed from its $\ell^{+} \ell^{-}$decays. A partial correction for final state radiation and bremsstrahlung energy loss is performed by including the four-momentum of every photon detected within a $50 \mathrm{mrad}$ cone around the electron direction in the $e^{+} e^{-}$invariant mass calculation.

The recoil mass spectrum of $J / \psi$ in inclusive $e^{+} e^{-} \rightarrow J / \psi X$ for the data is shown in Fig. 4. In addition to the three previously observed peaks, the $\eta_{c}, \chi_{c 0}, \eta_{c}(2 S)$, another significant peak can be seen around a mass of $3.94 \mathrm{GeV} / \mathrm{c}^{2}$.

A fit to this spectrum that includes three known $\left(\eta_{c}, \chi_{c 0}, \eta_{c}(2 S)\right)$ and one new $(X(3940))$ charmonium states is performed. In this fit, the mass positions for the $\eta_{c}, \chi_{c 0}$, $\eta_{c}(2 S)$ and $X(3940)$ are free parameters. The signal function for the $X(3940)$ is a convolution of the Monte Carlo line shape, with assumed zero width, with a Breit-Wigner function. The background is parametrized by a second order polynomial function and a threshold term $\left(\sqrt{M_{\text {recoil }}(J / \psi)-2 M_{D}}\right)$ to account for a possible contribution from $e^{+} e^{-} \rightarrow J / \psi D^{(*)} \bar{D}^{(*)}$. The mass of $X(3940)$ is measured to be $3.936 \pm 0.006 \pm 0.006$ $\mathrm{GeV} / \mathrm{c}^{2}$ and the width less than $52 \mathrm{MeV} / \mathrm{c}^{2}$ at $90 \%$ C.L.. The statistical significance of the signal is $5 \sigma$.

A search for $X(3940)$ decaying into $D \bar{D}$ and $D^{*} \bar{D}$ final states is performed. The decay $X(3940) \rightarrow D^{*} \bar{D}$ is found to be the dominant decay mode with a measured branching fraction of $96_{-32}^{+45} \pm 22 \%$. The upper limits for the decay $X(3940) \rightarrow D \bar{D}$ and $X(3940) \rightarrow J / \psi \omega$ are set to be less than $41 \%$ and $26 \%$ at $90 \%$

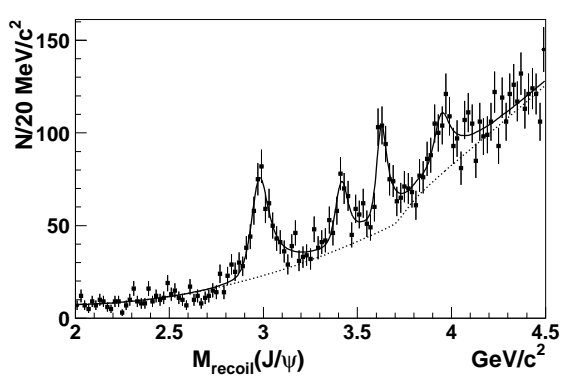

Figure 4. The distribution of masses recoiling against the reconstructed $J / \psi$ in inclusive $e^{+} e^{-} \rightarrow J / \psi X$ events. The enhancements correspond to the $\eta_{c}, \chi_{c 0}$, $\eta_{c}(2 S)$ and a new state, $X(3940)$. The curve represents the fit.

\section{C.L., respectively.}

\subsection{Observation of $Y(4260)$ in the $J / \psi \pi^{+} \pi^{-}$Mass Spectrum around $4.26 \mathrm{GeV} / \mathrm{c}^{2}$}

The initial-state radiation events, $e^{+} e^{-} \rightarrow$ $\gamma_{I S R} \pi^{+} \pi^{-} J / \psi$ are studied at BaBar, using an integrated luminosity of $211 \mathrm{fb}^{-1}$ data collected at $\sqrt{s}=10.58 \mathrm{GeV} / \mathrm{c}^{2}$, near the peak of the $\Upsilon(4 S)$ resonance and $22 f b^{-1}$ data collected approximately $40 \mathrm{MeV} / \mathrm{c}^{2}$ below this energy at the SLAC PEP-II asymmetricenergy $e^{+} e^{-}$storage ring. The candidate $J / \psi$ is reconstructed via its decay to $e^{+} e^{-}$and $\mu^{+} \mu^{-}$.

The $\pi^{+} \pi^{-} J / \psi$ invariant mass spectrum for candidates passing all criteria is shown in Fig. 5 as points with error bars. Events that have an $e^{+} e^{-}\left(\mu^{+} \mu^{-}\right)$mass in the $J / \psi$ sidebands $[2.76,2.95]$ or $[3.18,3.25]$ ([2.93, $3.01]$ or $[3.18,3.25]) \mathrm{GeV} / \mathrm{c}^{2}$ but pass all the other selection criteria are represented by the shaded histogram after being scaled by the ratio of the widths of the $J / \psi$ mass window and sideband regions. An enhancement near $4.26 \mathrm{GeV} / \mathrm{c}^{2}$ is clearly observed. The Fig. 5 inset includes the $\psi^{\prime}$ region with a logarithmic scale for comparison.

An unbinned likelihood fit to the $\pi^{+} \pi^{-} J / \psi$ mass spectrum is performed using a single relativistic Breit-Wigner signal 


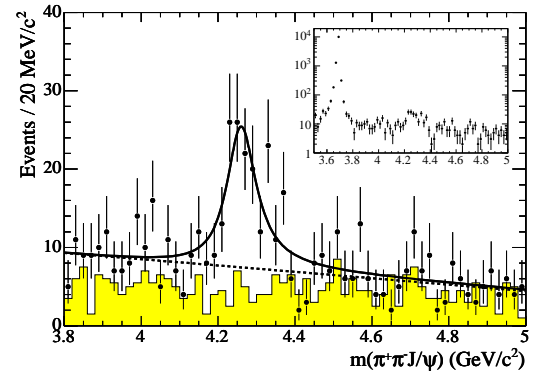

Figure 5. The $\pi^{+} \pi^{-} J / \psi$ invariant mass spectrum in the range $3.8-5.0 \mathrm{GeV} / \mathrm{c}^{2}$ and (inset) over a wider range that includes the $\psi^{\prime}$. The points with error bars represent the selected data and the shaded histogram represents the scaled data from neighboring $e^{+} e^{-}$and $\mu^{+} \mu^{-}$mass regions. The solid curve shows the result of the single-resonance fit; the dashed curve represents the background component.

function and a second-order polynomial background. The signal function is multiplied by a phase space factor and convoluted with a resolution function. The fit gives $125 \pm 23$ events with a mass of $4259 \pm 8(\text { stat })_{-6}^{+2}$ (syst) $\mathrm{MeV} / \mathrm{c}^{2}$ and a width of $88 \pm 23(\text { stat })_{-4}^{+6}$ (syst) $\mathrm{MeV} / \mathrm{c}^{2}$. The statistical significance of the signal is larger than $8 \sigma$. At the present level of the statistics, the existence of additional narrow resonance(s) in this region cannot be excluded. The broad stucture at around 4.26 $\mathrm{GeV} / \mathrm{c}^{2}$ signifies the presence of one or more previously unobserved $J^{P C}=1^{--}$states containing hidden charm.

\section{Heavy (flavor) quarkonium production}

There are many challenging problems remaining unsolved in heavy quarkonium physics. The effective field theory NRQCD factorization approach provides a systematic method for calculating quarkonium decay and production rates. NRQCD has been very successful in describing many production data, such as the inclusive $\mathrm{P}$-wave quakonium decays, quarkonium production at Tevatron and quarkonium production in deep inelastic scattering at HERA. However it is problematic in describing the quarkonium polarization data at the Tevatron, as well as the data from double charmonium production at $\mathrm{B}$ factories. One of the most challenging open problems in heavy quarkonium is the large discrepancy of the double charmonium production cross sections measured in $e^{+} e^{-}$annihilation at $\mathrm{B}$ factories and the theoretical calculations from NRQCD. Here, I'll only focus on this topic.

Belle $^{14}$ observed the double $c \bar{c}$ production in $e^{+} e^{-}$annihilation at $\sqrt{s} \sim 10.6 \mathrm{GeV}$. The production cross sections measured by Belle are about one order of magnitude higher than those predicted by non-relativistic QCD (NRQCD) calculations ${ }^{15,16,17}$ for $e^{+} e^{-} \rightarrow$ $\gamma^{*} \rightarrow J / \psi c \bar{c}$ reactions, where $c \bar{c}$ is a charmonium state with even C-parity. There have been attempts ${ }^{18,19,20,21,22}$ to reconcile the large discrepancy between the observed cross section and predictions, and the validity of NRQCD approximations has been questioned $^{23,24}$. It has also been suggested that at least part of the double charmonium production might be due to two virtualphoton interactions ${ }^{20}$, i.e., $e^{+} e^{-} \rightarrow \gamma^{*} \gamma^{*} \rightarrow$ $J / \psi c \bar{c}$, where odd C-parity states could be produced. However, Belle's updated results show that the contamination from $e^{+} e^{-} \rightarrow$ $\gamma \gamma \rightarrow J / \psi J / \psi$ is small ${ }^{25}$.

Recently, Belle and Babar presented the new measurements of the cross sections for double charmonium productions, using their $155 \mathrm{fb}^{-1}$ and $124 \mathrm{fb}^{-1}$ data taken at around $\Upsilon(4 S)$ peak, respectively.

The recoil mass distribution for events in the $J / \psi$ mass window is shown as points with error bars in Fig. 6. The upper plot is from Belle and the lower from BaBar. The fits to the recoil mass distribution are represented by the solid curves and the backgrounds are shown as the dashed curves.

The fit results are given in Table 1. The cross sections measured by both experiments are much larger than those predicted by many NRQCD calculations. 

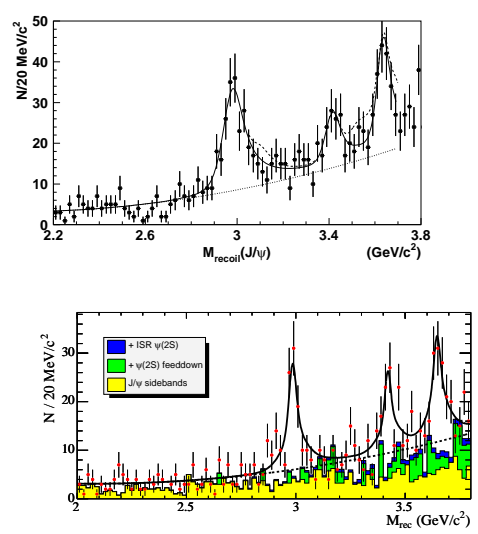

Figure 6. The points with error bars refer to the events in the $J / \psi$ mass window. The solid curves represent the fit and the dashed curves are for background. The upper plot is for Belle and the lower for Babar.

Table 1. Comparison of experimental cross-sections with theoretical expectations $(f b)$.

\begin{tabular}{ccc}
\hline \hline$J / \psi c \bar{c}$ & $\eta_{c}$ & $\chi_{c 0}$ \\
\hline BaBar & $17.6 \pm 2.8_{-2.1}^{+1.5}$ & $10.3 \pm 2.5_{-1.8}^{+1.4}$ \\
Belle & $25.6 \pm 2.8 \pm 3.4$ & $6.4 \pm 1.7 \pm 1.0$ \\
NRQCD $^{16}$ & $2.31 \pm 1.09$ & $2.28 \pm 1.03$ \\
NRQCD $^{15}$ & 5.5 & 6.9 \\
\hline \hline
\end{tabular}

\section{Heavy hadron spectroscopy}

\section{1 (Re)discovery of $\eta_{c}(2 S)$ and $h_{c}(1 P)$ states}

The two $c \bar{c}$ states that are expected to be below open charm threshold are still not well established. They are the radially excited $n=2$ singlet $S$ state, the $\eta_{c}(2 S)$ meson, and the $n=1$ singlet $P$ state, the $h_{c}(1 P)$. The observation of these states and the determination of their masses would complete the below-threshold charmonium particle spectrum and provide useful information about the spin-spin part of the charmonium potential.

In 1982, the Crystal Ball collaboration ${ }^{26}$ reported the observation of a small enhancement at $E_{\gamma} \sim 91 \mathrm{MeV}$ in the inclusive photon spectrum from $\psi^{\prime} \rightarrow \gamma X$ decays, and interpreted it as due to $\eta_{c}(2 S)$ with mass
Table 2. The measured $\eta_{c}(2 S)$ mass

\begin{tabular}{ccc}
\hline \hline Exp. & reactions & Mass $\left(\mathrm{MeV} / \mathrm{c}^{2}\right)$ \\
\hline Belle(02) & $B \rightarrow K K_{s} K \pi$ & $3654 \pm 6 \pm 8$ \\
Belle(03) & $e^{+} e^{-} \rightarrow J / \psi X$ & $3622 \pm 12$ \\
BaBar(03) & $\gamma \gamma \rightarrow K_{s} K^{+} \pi^{-}$ & $3630.8 \pm 3.4 \pm 1.0$ \\
CLEO $(03)$ & $\gamma \gamma \rightarrow K_{s} K^{+} \pi^{-}$ & $3642.9 \pm 3.1 \pm 1.5$ \\
\hline Average & & $3637.7 \pm 4.4$ \\
\hline \hline
\end{tabular}

$3594 \pm 5 \mathrm{MeV} / c^{2}$. This result implies a $\psi(2 S)$ $\eta_{c}^{\prime}$ mass splitting that is considerably larger than heavy-quark potential model expectations. The result was not confirmed by other experiments ${ }^{27}$.

The Belle experiment reported their observation of the $\eta_{c}(2 S)$ in exclusive $B \rightarrow$ $K K_{s} K \pi$ decays in $2002^{28}$ and then in double charmonium production process $e^{+} e^{-} \rightarrow$ $J / \psi X^{29}$. Later, both $\mathrm{BaBar}^{30}$ and $\mathrm{CLEO}^{31}$ confirmed the observation of $\eta_{c}(2 S)$ in $\gamma \gamma \rightarrow$ $K_{s} K^{+} \pi^{-}$reaction. The measured masses of $\eta_{c}(2 S)$ are listed in Table 2. Recent four measurements give consistent results on $\eta_{c}(2 S)$ mass, and the averaged mass of $\eta_{c}(2 S)$ from these experiments is significantly higher than that reported by Crystal Ball Collaboration. The deduced mass splitting of $m_{\psi(2 S)}-m_{\eta_{c}(2 S)}=48.4 \pm 4.5 \mathrm{MeV} / \mathrm{c}^{2}$ is consistent with the heavy quark potential model calculations $\left(42-103 \mathrm{MeV} / \mathrm{c}^{2}\right)^{32}$ and recent quenched LQCD calculations (40 -74 $\left.\mathrm{MeV} / \mathrm{c}^{2}\right)^{33}$.

As mentioned above, QCD-based potential models have been quite successful in predicting masses, widths, and dominant decays of several charmonium states. The theoretical calculations also made the prediction that the hyperfine splitting $\Delta M_{h f}\left(\left\langle M\left({ }^{3} P_{J}\right)\right\rangle-\right.$ $\left.M\left({ }^{1} P_{1}\right)\right)$ for $P$-wave states should be zero. Higher-order corrections are expected to provide no more than a few $\mathrm{MeV}$ deviation from this result ${ }^{34,35}$. Lattice QCD calculations ${ }^{33}$ predict $\Delta M_{h f}(1 P)=+1.5$ to $+3.7 \mathrm{MeV}$, but with uncertainties at the few-MeV level. Larger values of $\Delta M_{h f}(1 P)$ could result if the confinement potential had a vector component or if coupled channel effects were im- 
portant. In order to discriminate between these possibilities, it is necessary to identify the $h_{c}\left({ }^{1} P_{1}\right)$ state and to precisely measure its mass.

About 20 years ago, R704 experiment at CERN observed a cluster of 5 events in an exclusive scan for $p \bar{p} \rightarrow J / \psi+X^{36}$. The mass of this cluster is $3525.4 \pm 0.8 \mathrm{MeV} / \mathrm{c}^{2}$.

The Crystal Ball Collaboration searched for $h_{c}$ in the reaction $\psi(2 S) \rightarrow \pi^{0} h_{c}, h_{c} \rightarrow$ $\gamma \eta_{c}$, but did not see it in the mass range $M\left(h_{c}\right)=(3515-3535) \mathrm{MeV}^{37}$. The FNAL E760 Collaboration searched for $h_{c}$ in the reaction $p \bar{p} \rightarrow h_{c} \rightarrow \pi^{0} J / \psi, J / \psi \rightarrow e^{+} e^{-}$, and reported a statistically significant enhancement with $M\left(h_{c}\right)=3526.2 \pm 0.2 \pm 0.2$ $\mathrm{MeV}, \Gamma\left(h_{c}\right) \leq 1.1 \mathrm{MeV}^{38}$. The measurement was repeated twice by the E835 experiment with $\sim 2 \times$ and $\sim 3 \times$ larger luminosity, but no confirming signal for $h_{c}$ was observed in $h_{c} \rightarrow \pi^{0} \mathrm{~J} / \psi$ decay $^{39}$. E835 experiment also searched for $h_{c}$ state by a scan of $p \bar{p}$ annihilation cross section for $p \bar{p} \rightarrow h_{c} \rightarrow \gamma \eta_{c} \rightarrow \gamma \gamma \gamma$. An excess of $\eta_{c} \gamma$ events is observed, with a mass of $M=3525.8 \pm 0.2 \pm 0.2 \mathrm{MeV}$. It has a probability $\mathcal{P} \sim 0.001$ to arise from background fluctuations ${ }^{40}$.

Using $3.08 \times 10^{6} \psi(2 S)$ events accumulated with CLEO III and CLEO-c detector at the Cornell Electron Storage Ring, the isospin-violating reaction $e^{+} e^{-} \rightarrow \psi(2 S) \rightarrow$ $\pi^{0} h_{c}, h_{c} \rightarrow \gamma \eta_{c}, \pi^{0} \rightarrow \gamma \gamma$ is studied in exclusive and inclusive $\eta_{c}$ decays, and $h_{c}$ signal is observed in both cases. The statistical significance of $h_{c}$ is larger than $5 \sigma$ using a variety of methods to evaluate this quantity.

Table 3 shows the results for inclusive and exclusive analyses for the decay $\psi(2 S) \rightarrow$ $\pi^{0} h_{c} \rightarrow \pi^{0} \gamma \eta_{c}$. The combined inclusive and exclusive results give $M\left(h_{c}\right)=3524.4 \pm 0.6 \pm$ $0.4 \mathrm{MeV}$, which is consistent with the spinweighted average of the $\chi_{c J}$ states.
Table 3. Results of the inclusive and exclusive analyses for the reaction $\psi(2 S) \rightarrow \pi^{0} h_{c} \rightarrow \pi^{0} \gamma \eta_{c}$.

\begin{tabular}{lcc}
\hline \hline & Inclusive & Exclusive \\
\hline Counts & $150 \pm 40$ & $17.5 \pm 4.5$ \\
Significance & $\sim 3.8 \sigma$ & $6.1 \sigma$ \\
$M\left(h_{c}\right)(\mathrm{MeV})$ & $3524.9 \pm 0.7 \pm 0.4$ & $3523.6 \pm 0.9 \pm 0.5$ \\
$\mathcal{B}_{\psi} \mathcal{B}_{h}\left(10^{-4}\right)$ & $3.5 \pm 1.0 \pm 0.7$ & $5.3 \pm 1.5 \pm 1.0$ \\
\hline \hline
\end{tabular}

\section{2 $X(3872)$}

The $X(3872)$ was first observed by Belle ${ }^{41}$ in $B^{ \pm} \rightarrow K^{ \pm}\left(J / \psi \pi^{+} \pi^{-}\right)$and then confirmed by $\mathrm{CDF}^{42}, \mathrm{D}^{43}$ and $\mathrm{BaBar}^{44}$ experiments. The four experiments give consistent mass values of $X(3872)$. The averaged mass of $X(3872)$ is $3871.9 \pm 0.6 \mathrm{MeV} / \mathrm{c}^{2}$, which is just above $D^{0} \bar{D}^{* 0}$ threshold $(3871.3 \pm 1.0$ $\left.\mathrm{MeV} / \mathrm{c}^{2}\right)$.

Numerous theoretical explanations have been proposed for this high-mass, narrowwidth state decaying into $J / \psi \pi^{+} \pi^{-}$. The possibilities include a $c \bar{c}$ charmonium state ${ }^{45}$, $D^{0} \bar{D}^{* 0}$ molecular state ${ }^{46}$, dominant $1^{++}(2 P)$ $c \bar{c}$ component with $D^{0} \bar{D}^{* 0} / D^{* 0} \bar{D}^{0}$ continuum component ${ }^{47}, c \bar{c} g$ hybrid ${ }^{48}$, vector glueball with a small mixture of $c \bar{c}^{49}, S$-wave threshold enhancement in $D^{0} \bar{D}^{* 0}$ scattering ${ }^{50}$ and diquark-diquark bound state $c u \bar{c} \bar{u}^{51}$. The search for more decay modes of $X(3872)$ as well as the determination of its $J^{P C}$ will be helpful in understanding the nature of $X(3872)$

A more detailed examination of the $X(3872)$ indicates that the $\pi^{+} \pi^{-}$mass distributions peak near the kinematic upper limit and are consistent with the decay $\rho^{0} \rightarrow$ $\pi^{+} \pi^{-}$. If the observed decay is $X(3872) \rightarrow$ $J / \psi \rho^{0}$ and if these states and their decays obey isospin symmetry, then there must be a $X(3872)^{-}$, which decays to $J / \psi \rho^{-}$, and the rate for $B \rightarrow X^{-} K$ should be twice of that for $B \rightarrow X^{0} K$. For this purpose, BaBar has performed a search for the decays of $B^{0} \rightarrow X^{-} K^{+}$and $B^{-} \rightarrow X^{-} K_{s}$, where $X^{-} \rightarrow J / \psi \pi^{-} \pi^{052}$, using $234 \mathrm{M} B \bar{B}$ events. No charged signal, $X^{-} \rightarrow J / \psi \pi^{-} \pi^{0}$, is evi- 
dent at $3.872 \mathrm{GeV} / \mathrm{c}^{2}$.

BaBar also searched for $X(3872) \rightarrow$ $\eta J / \psi$ with $90 \mathrm{M} B \bar{B}$ events and no signal is seen $^{53}$.

CLEO searched for $X(3872)$ in $\gamma \gamma \mathrm{fu}-$ sion and radiative production data and no evidence is found ${ }^{54}$.

In order to examine the experimental constraints on the possible $J^{P C}$ of $X(3872)$, Belle searched for $B \rightarrow K \gamma J / \psi$ and $K \pi^{+} \pi^{-} \pi^{0} J / \psi$ decays in a $275 \mathrm{M} B \bar{B}$ events sample, accumulated at a center-of-mass system energy of $\sqrt{s}=10.58 \mathrm{GeV}$. In the $\gamma J / \psi$ mass spectrum, a peak around $3872 \mathrm{MeV} / \mathrm{c}^{2}$ can be seen, which corresponds to a statistical significance of $4.0 \sigma$. The ratio of the decay widths is obtained from the fit as:

$\Gamma(X \rightarrow \gamma J / \psi) / \Gamma\left(X \rightarrow \pi^{+} \pi^{-} J / \psi\right)=0.14 \pm 0.05$

The $\pi^{+} \pi^{-} \pi^{0}$ mass spectrum in $B \rightarrow$ $\pi^{+} \pi^{-} \pi^{0} J / \psi$ also shows the evidence for subthreshold decay $X(3872) \rightarrow \omega J / \psi$ and the statistical significance is $4.3 \sigma$. The ratio of the branching fractions is determined to be:

$$
\frac{B\left(X \rightarrow \pi^{+} \pi^{-} \pi^{0} J / \psi\right)}{B\left(X \rightarrow \pi^{+} \pi^{-} J / \psi\right)}=1.0 \pm 0.4 \pm 0.3 .
$$

The evidence of these two decay modes suggests the $C$ parity of $X(3872)$ to be +1 .

In a search for $X(3872) \rightarrow D^{0} \bar{D}^{* 0}$ decay in $B \rightarrow K D^{0} \bar{D}^{* 0}$, with $\bar{D}^{* 0} \rightarrow \bar{D}^{0} \pi^{0}$, there is events excess at around $3872 \mathrm{MeV} / \mathrm{c}^{2}$ in $D^{0} \bar{D}^{0} \pi^{0}$ spectrum. This implies that the $2^{++}$ is not favored.

The angular distributions of a sample of $X(3872)$, produced in $B \rightarrow K X(3872)$ from $256 \mathrm{pb}^{-1}$ data are analyzed. From the $\chi^{2}$ test, a $1^{++} X(3872)$ is favored.

All the results seem to favor a $1^{++}$ $X(3872)$. However, the statistics is low for the evidences of $X(3872) \rightarrow \gamma J / \psi$, $X(3872) \rightarrow \omega J / \psi$ and $X(3872) \rightarrow D^{0} \bar{D}^{* 0}$. In the analyses of the angular distributions, the statistics is low to use $\chi^{2}$ test. Therefore, more data are needed and the confirmation from BaBar is required to finally determine the spin-parity of $X(3872)$.

\section{Selected topics from quarkonium decays}

\subsection{The $\sigma$ production}

There has been evidence for a low mass pole in the early $\mathrm{DM}^{55}$ and $\mathrm{BESI}^{56}$ data on $J / \psi \rightarrow \omega \pi^{+} \pi^{-}$. A huge event concentration in the $I=0$ S-wave $\pi \pi$ channel was seen in the region of $m_{\pi \pi}$ around 500-600 $\mathrm{MeV}$ in a $p p$ central production experiment ${ }^{57}$. This peak is too large to be explained as background $^{58}$. There have been many studies on the possible resonance structure in $\pi \pi$ elastic scattering ${ }^{59}$. It was later proved that the $\sigma$ resonance is unavoidable in chiral perturbation theory in order to explain the $\pi \pi$ scattering phase shift data ${ }^{60}$. E791 experiment from FNAL shows the existence of a $\sigma$ pole with $M=478_{-23}^{+24} \pm 17 \mathrm{MeV}$, $\Gamma=324_{-40}^{+42} \pm 21 \mathrm{MeV}$ in $D^{+} \rightarrow \pi^{+} \pi^{-} \pi^{+}$ 61.

BES studied $J / \psi \rightarrow \omega \pi^{+} \pi^{-}$decays based on $58 \mathrm{M} J / \psi$ events collected at BESII. Figure 7 shows the $\pi^{+} \pi^{-}$invariant mass spectrum recoiling against $\omega$. In addition to the well known $f_{2}(1270)$, a broad bump in the low mass region is clearly seen. Two independent partial wave analyses (PWA) are performed and different parametrizations of $\sigma$ amplitude are used. All give the consistent results for $\sigma$ pole position. The averaged pole is determined to be $(541 \pm 39-i(252 \pm 42))$ $\mathrm{MeV} / \mathrm{c}^{2}$.

Based on $14 \mathrm{M} \psi(2 S)$ events collected at BESII, a partial wave analysis is performed to $\psi(2 S) \rightarrow \pi^{+} \pi^{-} J / \psi$, with $J / \psi \rightarrow \mu^{+} \mu^{-}$. A severe suppression of the $\pi^{+} \pi^{-}$invariant mass near the $\pi \pi$ threshold is distinctively different from the phase space shape, which suggests the $\sigma$ production. Using different parametrizations of $\sigma$ amplitude, the data can be fitted well through a strong cancellation between $\sigma$ and a contact term. The obtained pole posision is $(554 \pm 14 \pm 53)-i$ $(242 \pm 5 \pm 24)) \mathrm{MeV} / \mathrm{c}^{2}$, which is consistent with that from $J / \psi \rightarrow \omega \pi^{+} \pi^{-}$. 


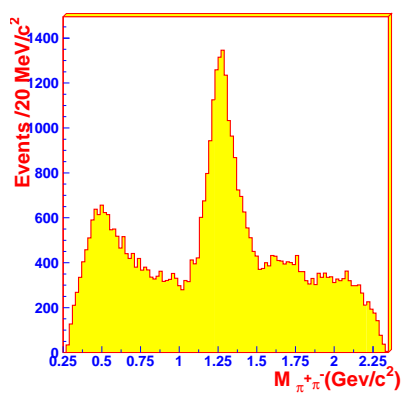

Figure 7 . The $\pi^{+} \pi^{-}$invariant mass recoiling against $\omega$

BaBar made a full amplitude analysis on $B^{ \pm} \rightarrow \pi^{ \pm} \pi^{ \pm} \pi^{\mp}$ with $210.3 f b^{-1}$ data sample and found that the decays of $B^{ \pm} \rightarrow \pi^{ \pm} \pi^{ \pm} \pi^{\mp}$ are dominated by decays through the intermediate resonances, such as $\rho^{0}(770)$ and $\rho^{0}(1450)$. There is no evidence for the exisitence of $\sigma$.

\subsection{The $\kappa$ production}

The $\kappa$ has been observed in the analyses on $K \pi$ scattering phase shifts by several groups. The observed mass and width are scattered in the ranges from 700 to $900 \mathrm{MeV} / \mathrm{c}^{2}$ and 550 to $800 \mathrm{MeV} / \mathrm{c}^{2}$, respectively, depending on the model used. However, some analyses on $K \pi$ scattering data don't need a $\kappa$ pole. The E791 experiment at Fermilab reported the evidence of $\kappa$ in the $D^{+} \rightarrow K^{+} \pi^{+} \pi^{-62}$ with the mass and width of $797 \pm 19 \pm 43 \mathrm{MeV} / \mathrm{c}^{2}$ and $410 \pm 43 \pm 87 \mathrm{MeV} / \mathrm{c}^{2}$. However, a slightly lower statistics of CLEO $D^{0} \rightarrow K^{-} \pi^{+} \pi^{0}$ data finds no evidence of $\kappa^{63}$. The FOCUS experiment presented evidence for the existence of a coherent $K \pi S$-wave contribution to $D^{+} \rightarrow K^{-} \pi^{+} \mu^{+} \nu^{64}$.

BES performed partial wave analyses to both $J / \psi \rightarrow K^{*} K \pi^{65}$ and direct $J / \psi \rightarrow$ $K^{+} K^{-} \pi^{+} \pi^{-}$processes. The $\kappa$ are needed in both fits. Fig. 8 is the $K \pi$ invariant mass spectrum which recoils against $K^{*}(892)$. The crosses are data and histograms represent the PWA fit projection. The shaded area

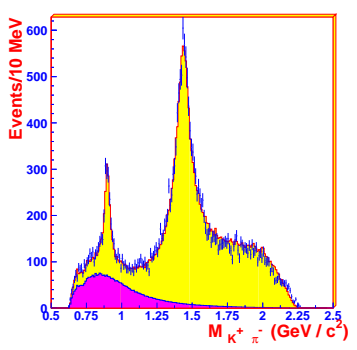

Figure 8 . The $K \pi$ invariant mass recoiling against $K^{*}$. The crosses are data and histograms represent the PWA fit projection. The shaded area shows the $\kappa$ contribution.

shows the $\kappa$ contribution. The pole of $\kappa$ from $J / \psi \rightarrow K^{*} K \pi$ is determined to be $\left(841 \pm 30_{-73}^{+81}\right)-i\left(309 \pm 45_{-72}^{+48}\right) \mathrm{MeV} / \mathrm{c}^{2}$.

Both BaBar and Belle made the Dalitz plot analyses of $B \rightarrow K \pi \pi$, but no $\kappa$ is included in the fit ${ }^{66}$.

\section{$5.3 \psi(3770)$ non $-D \bar{D}$ decays}

$\psi(3770)$ was considered to decay almost entirely to pure $D \bar{D}^{67}$ because its mass is above the open charm-pair threshold and its width is two orders of magnitude larger than that of $\psi(2 S)$. Since $\psi(3770)$ is also believed to be a mixture of the $1^{3} D_{1}$ and $2^{3} S_{1}$ states $^{68}$, other $\psi(2 S)$-like decays for $\psi(3770)$ are expected. Many theoretical calculations ${ }^{69,70,71,72,73}$ estimated the partial width of $\Gamma(\psi(3770) \rightarrow$ $\pi \pi J / \psi)$. Recently, Kuang ${ }^{73}$ obtained the partial width for $\psi(3770) \rightarrow J / \psi \pi^{+} \pi^{-}$to be in the range of 25 to $113 \mathrm{keV}$, using ChenKuang potential model.

Based on $27.7 \mathrm{pb}^{-1}$ data sample taken in the center-of-mass (c.m.) energy region of $3.738 \mathrm{GeV}$ to $3.885 \mathrm{GeV}$, BES reported the first evidence of $\psi(3770) \rightarrow J / \psi \pi^{+} \pi^{-}$ with $J / \psi \rightarrow e^{+} e^{-}$and $\mu^{+} \mu^{-74}$. Figure 9 shows the dilepton masses determined from the fitted lepton momenta of the accepted events. Two peaks are clearly seen. The lower peak mostly comes from $\psi(3770) \rightarrow$ $J / \psi \pi^{+} \pi^{-}$, while the higher one is produced by the radiative return to the $\psi(2 S)$ peak. 


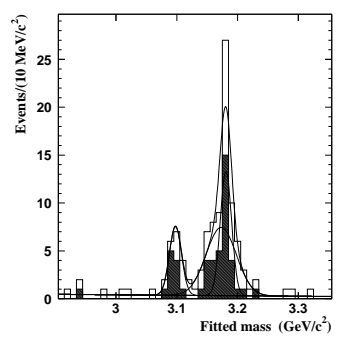

Figure 9. The distribution of the fitted dilepton masses for the events of $l^{+} l^{-} \pi^{+} \pi^{-}$from the data; the hatched histogram is for $\mu^{+} \mu^{-} \pi^{+} \pi^{-}$, while the open one is for $e^{+} e^{-} \pi^{+} \pi^{-}$; the curves give the best fit to the data.

A maximum likelihood fit gives $17.8 \pm 4.8$ $J / \psi \rightarrow l^{+} l^{-}$signal events. After subtracting the background, $(11.8 \pm 4.8 \pm 1.3) \psi(3770) \rightarrow$ $J / \psi \pi^{+} \pi^{-}$non- $D \bar{D}$ decay events are observed, leading to a branching fraction of $B F\left(\psi(3770) \rightarrow J / \psi \pi^{+} \pi^{-}\right)=(0.34 \pm 0.14 \pm$ $0.09) \%$, and a partial width $\Gamma(\psi(3770) \rightarrow$ $\left.J / \psi \pi^{+} \pi^{-}\right)=(80 \pm 33 \pm 23) \mathrm{keV}$, which is consistent with Kuang's estimations ${ }^{73}$.

With a much larger data sample (an integrated luminosity of $\mathcal{L}=(280.7 \pm$ $2.8 \mathrm{pb}^{-1}$ ), CLEO confirmed the non- $D \bar{D}$ decays of $\psi(3770) \rightarrow \pi \pi J / \psi$ with the statistical significancies of $13 \sigma$ and $3.8 \sigma$ for $\pi^{+} \pi^{-}$ and $\pi^{0} \pi^{0}$ cases, respectively ${ }^{75}$. More precise branching fractions are obtained to be: $B F\left(\psi(3770) \rightarrow J / \psi \pi^{+} \pi^{-}\right)=(0.214 \pm 0.25 \pm$ $0.22) \%$ and $B F\left(\psi(3770) \rightarrow J / \psi \pi^{0} \pi^{0}\right)=$ $(0.097 \pm 0.035 \pm 0.020) \%$. The partial widthes are $\Gamma\left(\psi(3770) \rightarrow J / \psi \pi^{+} \pi^{-}\right)=(50 \pm 6 \pm$ $8) \mathrm{keV}$ and $\Gamma\left(\psi(3770) \rightarrow J / \psi \pi^{0} \pi^{0}\right)=(23 \pm$ $8 \pm 5) \mathrm{keV}$. The $\psi(3770) \rightarrow J / \psi \pi^{+} \pi^{-}$results are consistent with those from BES but with higher precision.

Using the same data sample, CLEO first observed $\psi(3770) \rightarrow \gamma \chi_{c 1} \rightarrow \gamma \gamma J / \psi$, with $J / \psi \rightarrow \mu^{+} \mu^{-}$and $e^{+} e^{-76}$. Figure 10 shows the energy of the lower energy photon for the selected $\psi(3770) \rightarrow \gamma \gamma J / \psi, J / \psi \rightarrow \mu^{+} \mu^{-}$ (top) and $J / \psi \rightarrow e^{+} e^{-}$(bottom). The excess in the $\chi_{c 1}$ peak represents evidence for $\psi(3770) \rightarrow \gamma \chi_{c 1}$ transitions. The fitted sig-

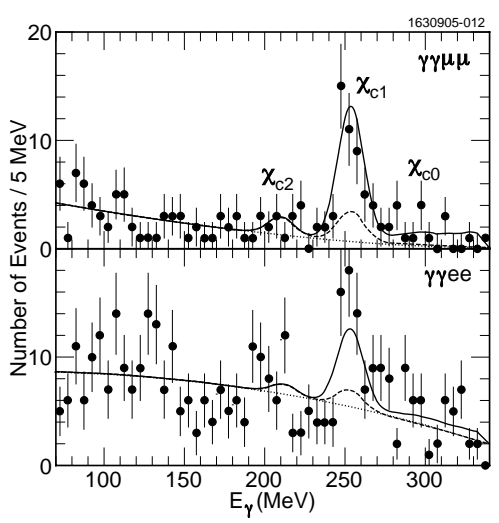

Figure 10. Energy of the lower energy photon for the selected $e^{+} e^{-} \rightarrow \gamma \gamma J / \psi, J / \psi \rightarrow \mu \mu$ (top) and $J / \psi \rightarrow e^{+} e^{-}$(bottom) events at the $\psi(3770)$ resonance. The solid line shows the fit. The dotted line shows the smooth background. The dashed line shows the total background including the expected background-peaks from radiatively produced tail of the $\psi(2 S)$ resonance.

nal amplitudes (for the sum of the dimuon and dielectron samples) are $0.3_{-0.3}^{+7.7}, 62 \pm 11$ and $24_{-15}^{+12}$ events for $\chi_{c 2}, \chi_{c 1}$ and $\chi_{c 0}$, respectively. The statistical significance of the evidence for $\chi_{c 1}$ signal is $6.8 \sigma$ and the decay width of $\psi(3770)$ to $\gamma \chi_{c 1}$ is $75 \pm 14 \pm 13(\mathrm{keV})$.

Both CLEO and BES also performed many searches for $\psi(3770)$ non$D \bar{D}$ decays ${ }^{77,78,79,80}$. A statistically significant signal is found for $\phi \eta^{77}$ by CLEO and a suggestive suppression of $\pi^{+} \pi^{-} \pi^{0}$ and $\rho \pi$ is observed by CLEO and $\operatorname{BES}^{77,79}$.

\section{Summary}

Many new discoveries and new results on heavy quarkonium productions and decays have been recently experimentally revitalized by BES, Belle, BaBar, CLEO, as well as other experiments. This report represents the results including the new observed particles, such as $X(1835), X(3940), Y(3940)$ and $Y(4260)$, further studies of $X(3872)$, the observation of $h_{c}$ state, heavy flavor production, in particular the double charmonium production from BaBar and Belle, the production of 
$\sigma$ and $\kappa$, as well as $\psi(3770)$ non- $D \bar{D}$ decays.

More results on quarkonium are comming in the near future from BESII, CLEO-c, Belle, Babar and the experiments from Fermilab. The BESIII/BEPCII is being constructing and it will start taking physics data in the year of 2008 . There is also a proposal for a new dedicated $p \bar{p}$ machine (PANDA at GSI) to explore charmonium physics. We expect more results on quarkonium production and decay to probe the strong interactions.

\section{Acknowledgments}

I would like to thank many colleagues from BaBar, Belle, BES, CLEO and the experiments at FNAL for providing me with information contained in this report. I apologize for not being able to discuss all the recent experimental results related to this talk due to the length limitation. I would also like to thank Prof. K.T. Chao and Y.P. Kuang for discussions concerning theoretical aspects.

\section{References}

1. I.S. Shapiro,Phys. Rept. 35, 129 (1978); C.B. Dover, M. Goldhaber, Phys. Rev. D 15, 1997 (1977).

2. BES Collaboration, J.Z. Bai et al., Phys. Rev. Lett. 91, 022001 (2003).

3. A. Datta, P.J. O'Donnell, Phys. Lett. B567, 273 (2003); M.L. Yan et al., hep-ph/0405087; B. Loiseau, S. Wycech, hep-ph/0502127.

4. J. Ellis, Y. Frishman and M. Karliner, Phys. Lett. B566, 201 (2003); J.L. Rosner, Phys. Rev. D 68, 014004 (2003).

5. C.S. Gao and S.L. Zhu, Commun. Theor. Phys. 42, 844 (2004), hepph/0308205.

6. G.J. Ding and M.L. Yan,Phys. Rev. C 72, 015208 (2005).

7. B.S. Zou and H.C. Chiang, Phys. Rev. D 69, 034004 (2003).

8. A. Sibirtsev et al., Phys. Rev. D 71,
054010 (2005).

9. BES Collaboration, M. Ablikim et al., Phys. Rev. Lett. 93, 112002 (2004).

10. For recent reviews of this subject, see E. Klempt et al., Phys. Rep. 368, 119 (2002) and J-M. Richard, Nucl. Phys. Proc. Suppl. 86, 361 (2000).

11. Particle Data Group, S. Eidelman et al., Phys. Lett. B592, 1 (2004).

12. D. Horn and J. Mandula, Phys. Rev. D 17, 898 (1978).

13. F.E. Close, I. Dunietz, P.R. Page, S. Veseli and H. Yamamoto, Phys. Rev. D 57, 5653 (1987); G. Chiladze, A.F. Falk and A.A. Petrov Phys. Rev. D 58, 034013 (1988); and F.E. Close and S. Godfrey, Phys. Lett. B 574, 210 (2003).

14. Belle Collaboration, K. Abe et al, Phys. Rev. Lett. 89, 142001 (2002).

15. Kui-Yong Liu, Zhi-Guo He and KuangTa Chao, Phys. Lett. B 557, 45 (2003).

16. E. Braaten and J. Lee, Phys. Rev. D 67, 054007 (2003).

17. K. Hagiwara, E. Kou and C.-F. Qiao, Phys. Lett. B 570, 39 (2003).

18. Y. J. Zhang, Y. J. Gao and K. T. Chao, hep-ph/0506076.

19. J.P. Ma and Z.G. Si, Phys. Rev. D 70, 074007 (2004)

20. G. T. Bodwin, J. Lee and E. Braaten, Phys. Rev. D 67, 054023 (2003).

21. G. T. Bodwin, J. Lee and E. Braaten, Phys. Rev. Lett. 90, 162001 (2003).

22. S. J. Brodsky, A. S. Goldhaber and J. Lee, Phys. Rev. Lett. 91, 112001 (2003).

23. A. E. Bondar and V. L. Chernyak, Phys. Lett. B 612, 215 (2005).

24. N. Brambilla et al, hep-ph/0412158.

25. Belle Collaboration, K. Abe et al, Phys. Rev. D 70, 071102 (2004).

26. C. Edwards et al (Crystal Ball Collab.), Phys. Rev. Lett. 48, 70 (1982).

27. T.A. Armstrong et al (E760 Collab.), Phys. Rev. D52, 4839 (1995); M. Ma- 
suzawa, Ph.D. Thesis, Northwestern Univ. report UMI-94-15774 (1993), unpublished; M. Ambrogiani et al., Phys. Rev. D64, 052003 (2001); P. Abreu et al. (DELPHI), Phys. Lett. B441, 479 (1998); and M.Acciarri et al. (L3), Phys. Lett. B461, 155 (1999).

28. Belle Collaboration, S.-K. Choi et al, Phys. Rev. Lett. 89 (2002) 102001; Erratum-ibid. 89129901 (2002).

29. Belle Collaboration, K. Abe et al, Phys. Rev. Lett. 89142001 (2002).

30. BaBar Collaboration, Phys. Rev. Lett. 92142002 (2004).

31. CLEO Collaboration, Phys. Rev. Lett. 92142001 (2004).

32. E. Eichten and F. Feinberg, Phys. Rev. D23, 2724 (1981); W. Buchmüller and S. -H. H. Tye, Phys. Rev. D24, 132 (1981); S. Godfrey and N. Isgur, Phys. Rev. D32, 189 (1985); Y. Q Chen and Y. P. Kuang, Phys. Rev. D37, 1210 (1988); E. J. Eichten and C. Quigg, Phys. Rev. D49, 5845 (1994); D. Ebert, R. N. Faustov and V. O. Galkin, Phys. Rev. D62, 034014 (2000).

33. K. Okamoto, Phys. Rev. D65, 094508 (2002)

34. T. Appelquist, R. M. Barnett and K. D. Lane, Ann. Rev. Nucl. Part. Sci. 28, 387 (1978).

35. S. Godfrey and J. L. Rosner, Phys. Rev. D 66, 014012 (2002).

36. R704 Collaboration, Phys. Lett. B 171, 135 (1986).

37. Proceedings of the 17th Rencontre de Moriond Workshop on New Flavors, Les Arcs, France, p. 27 (1982); E. D. Bloom and C. W. Peck, Ann. Rev. Nucl. Part. Sci., 33, 143 (1983).

38. E760 Collaboration, Phys. Rev. Lett., 69, 2337 (1992).

39. D. N. Joffe, Ph.D. thesis, Northwestern University, hep-ex/0505007

40. Claudia Patrignani, hep-ex/0410085

41. Belle Collaboration, Phys. Rev. Lett.,
91, 262001 (2003).

42. CDF-II Collaboration, Phys. Rev. Lett., 93, 072001 (2004).

43. D0 Collaboration, Phys. Rev. Lett., 93, 162002 (2004).

44. BaBar Collaboration, Phys. Rev. D 71, 071103 (2005).

45. E. Eichten, K. Lane, and C. Quigg, Phys. Rev. Lett. 89, 162002 (2002); T. Barnes and S. Godfrey, Phys. Rev. D 69, 054008 (2004).

46. N. Tornqvist, Phys. Lett. B 590, 209 (2004); M. B. Voloshin, Phys. Lett. $B$ 579, 316 (2004); F. Close and P. Page, Phys. Lett. B 578, 119 (2004); C.Y. Wong, Phys. Rev. C 69, 055202 (2004); E. Braaten and M. Kusunoki, Phys. Rev. D 69, 074005 (2004); E. Swanson, Phys. Lett. B 588, 189 (2004).

47. C. Meng, Y.J. Gao and K.T. Chao, hep$\mathrm{ph} / 0506222$

48. F. Close and S. Godfrey,Phys. Lett. B 574, 210 (2003) B.A. Li,Phys. Lett. $B$ 605, 306 (2005).

49. K.K. Seth, hep-ph/0411122

50. D.V. Bugg, Phys. Rev. D 71, 016006 (2005).

51. L. Maiani, F. Piccinini, A.D. Polosa, and V. Riquer, Phys. Rev. D 71, 014028 (2005).

52. BaBar Collaboration,Phys. Rev. Lett., 93, 041801 (2004).

53. BaBar Collaboration, Phys. Rev. D 71, 031501 (2005).

54. CLEO Collaboration, Phys. Rev. Lett., 94, 032004 (2005).

55. J.E. Augustin et al.,Nucl. Phys. B320 1 (1989)

56. Ning $\mathrm{Wu}$ (BES Collaboration), Proceedings of the XXXVIth Rencontres de Moriond, Les Arcs, France, March 1724, 2001 .

57. D. Alde et al.,Phys. Lett. B397 350 (1997).

58. T. Ishida et al., Proceedings of Int. Conf. Hadron'95, Manchester, UK, 
World Scientific, 1995.

59. V.E. Markushin and

M.P. Locher,Frascati Phys. Ser. 15229 (1999).

60. Z. Xiao, H. Q. Zheng, Nucl. Phys. A695 273 (2001).

61. E791 Collaboration, Phys. Rev. Lett. 86770 (2001).

62. E791 Collaboration, Phys. Rev. Lett. 89121801 (2002).

63. CLEO Collaboration, Phys. Rev. D63, 0900001 (2001)

64. FOCUS Collaboration,Phys. Lett. B535 430 (2002)

65. BES Collaboration, hep-ex/0506055

66. Belle Collaboration, Phys. Rev. D 71, 092003 (2005), BaBar Collaboration, BABAR-PUB-05/027

67. W. Bacino et al., DELCO Collaboration, Phys. Rev. Lett. 40671 (1978).

68. J.L. Rosner,Phys. Rev. D 64, 094002 (2001).

69. H.J. Lipkin,Phys. Lett. $\quad$ B179 278 (1986).

70. K. Lane, Harvard Report No. HUTP86/A045, (1986).

71. Y.P. Kuang and T.M. Yan, Phys. Rev. D 242874 (1981).

72. Y.P. Kuang and T.M. Yan, Phys. Rev. D 41155 (1990).

73. Y.P. Kuang, Phys. Rev. D 65094024 (2002).

74. BES Collaboration, hep-ex/0307028, Phys. Lett. B 605, 63 (2005).

75. CLEO collaboration, hep-ex/0508023

76. CLEO collaboration, hep-ex/0509030

77. CLEO collaboration, hep-ex/0509011

78. CLEO collaboration, CLNS 05/1921 (LP2005-443)

79. BES Collaboration, Phys. Rev. D70 077101 (2004).

80. BES Collaboration, hep-ex/0507092 


\section{DISCUSSION}

Ulrich Wiedner (Uppsala University):

Could you explain in more detail why the enhanced double charmonium production rate could not be explained by $J / \psi+$ glueball production?

Xiaoyan Shen: Belle ${ }^{1}$ measured the production and $J / \psi$ helicity angular distributions, $\left(1+\alpha_{\text {prod }} \cos ^{2} \theta_{\text {prod }}\right)$ and $(1+$ $\left.\alpha_{\text {heli }} \cos ^{2} \theta_{h e l i}\right)$, for $e^{+} e^{-} \rightarrow J / \psi \eta_{c}$. The $\alpha_{\text {prod }}$ and $\alpha_{\text {heli }}$ are measured to be: $\alpha_{\text {prod }}=\alpha_{\text {heli }}=0.93_{-0.47}^{+0.57}$. It is consistent with the expectations for the production of $J / \psi \eta_{c}$ via a single virtual photon $\left(\alpha_{\text {prod }}=\alpha_{\text {heli }}=1\right)$. The prediction for a spin-0 glueball contribution ${ }^{2}$ $\left(e^{+} e^{-} \rightarrow J / \psi G\right)$ to the $J / \psi \eta_{c}$ peak, $\alpha_{\text {prod }}=\alpha_{\text {heli }}=-0.87$, is disfavored.

\section{Tord Ekelof (Uppsala University):}

I have a question on the $\mathrm{X}(1834)$. You said that the $\mathrm{X}(1860)$ has $10 \%$ branching ratio to $\bar{p} p$ which was an evidence that it is a $\bar{p} p$ bound state. The $\mathrm{X}(1834)$ you discovered in this specific decay channel - I don't know how well these $10 \%$ can be put into a model but if this lower state the $\mathrm{X}(1834)$ should also then have some small branching ratio to $\bar{p} p$, how small would it be-would it be measurable?

Xiaoyan Shen: The large branching ratio of $X(1860), p \bar{p}$ threshold enhancement from $J / \psi \rightarrow \gamma p \bar{p}$, to $p \bar{p}$ indicates $X(1860)$ has a large coupling to $p \bar{p}$. If it is a $p \bar{p}$ bound state below $p \bar{p}$ mass threshold, the theory predicts that its $\eta^{\prime} \pi^{+} \pi^{-}$decay mode would be the most favorable one $\mathrm{e}^{3,4}$.

The mass and width of $X(1835)$ from $J / \psi \rightarrow \gamma \eta^{\prime} \pi^{+} \pi^{-}$are consistent with those of $X(1860)$. Therefore we think these two states could be the same state.
Jonathan L. Rosner (Uni. of Chicago): The $\Upsilon(4260)$ is just above $D_{s}^{*} \overline{D_{s}^{*}}$ threshold in the same way that the $\Upsilon(4030)$ is just above $D^{*} \overline{D^{*}}$ threshold. It might therefore be useful to look for $\Upsilon(4260) \rightarrow D_{s}^{*} \overline{D_{s}^{*}}$.

Xiaoyan Shen: It is a good suggestion. BaBar might be able to check it.

\section{References}

1. Belle Collaboration, K. Abe et al, Phys. Rev. D 70, 071102 (2004).

2. S. J. Brodsky, A. S. Goldhaber and J. Lee, Phys. Rev. Lett. 91, 112001 (2003).

3. C.S. Gao and S.L. Zhu, Commun. Theor. Phys. 42, 844 (2004), hepph/0308205.

4. G.J. Ding and M.L. Yan, Phys. Rev. C 72, 015208 (2005). 